\title{
Calidad de vida en pacientes con hiperhidrosis primaria sometidos a simpatectomía videotoracoscópica*
}

\author{
Drs. JAIME ZAMARÍN M. ${ }^{1}$, JOSEFINA EGAÑA D. ${ }^{1}$, RAÚL BERRÍOS S. ${ }^{1}$ \\ 1 Servicio de Cirugía, Hospital de la Fuerza Aérea de Chile. \\ Santiago, Chile.
}

\begin{abstract}
Quality of life after videothoracic simpathectomy in patients with primary hyperhidrosis

Introduction: Primary hyperhidrosis is characterized by excessive sweating that exceeds the physiological needs to maintain thermal homeostasis of the body. This study aims to assess the change in quality of life of patients operated on videothoracoscopic sympathectomy. Materials and Methods: We included all patients operated with this technique in the period between 2004 and 2010, in FACh Hospital. A standardized cuestionary was used to measure severity of hyperhidrosis and quality of life during pre and postoperative. Results: 61 patients, 57\% female and mean age of 25 years. Preoperative sweating was barely tolerable or unacceptable in $81 \%$, and preoperative quality of life was poor or very poor in $82 \%$ of patients. After surgery sweating was never noticed or tolerable at $85 \%$ and the quality of life was better in $84 \%$ of patients. $78 \%$ of patients developed compensatory sweating; however the $87 \%$ were satisfied with the outcome of surgery. Conclusion: Videothoracoscopic sympathectomy offers excellent results that are measurable in terms of improvement of quality of life, but often associated with the phenomenon of compensatory sweating. Despite the development of this sequel, the majorities of operated patients are satisfied with the surgical results and improve their quality of life.
\end{abstract}

Key words: Primary hyperhidrosis, sympathectomy, quality of life.

\section{Resumen}

Introducción: La hiperhidrosis primaria se caracteriza por sudoración excesiva que supera las necesidades fisiológicas para mantener la homeostasis térmica del cuerpo. El objetivo de este trabajo es evaluar el cambio en la calidad de vida de los pacientes operados de simpatectomía videotoracoscópica. Material y Método: Estudio de tipo transversal del total de pacientes operados con esta técnica en el Hospital Fuerza Aérea de Chile (FACh) en el período entre 2004 y 2010. Se utilizó una encuesta estandarizada y validada en la literatura para medir severidad de la hiperhidrosis y calidad de vida en período pre y postoperatorio. Resultados: 61 pacientes, 57\% de género femenino y promedio de edad 25 años. La sudoración preoperatoria

*Recibido el 23 de diciembre de 2010 y aceptado para publicación el 12 de marzo de 2011.

Correspondencia: Dr. Jaime Zamarín M.

Ricardo Cox 1575, Santiago, Chile.

jaimezamarin@hotmail.com 
era apenas tolerable o intolerable en $81 \%$ y la calidad de vida preoperatoria era pobre o muy pobre en $82 \%$ de los pacientes. Posterior a la cirugía la sudoración nunca se notaba o era tolerable en $85 \%$ y la calidad de vida era mejor en $84 \%$ de los pacientes. El 78\% de los pacientes operados desarrolló sudoración compensatoria, sin embargo, el $87 \%$ de los pacientes estaban satisfechos con el resultado de la cirugía. Conclusión: La simpatectomía videotoracoscópica ofrece excelentes resultados que son medibles en términos de mejoría de la calidad de vida, pero asociado frecuentemente al fenómeno de sudoración compensatoria. Pese al desarrollo de esta secuela, la mayoría de los pacientes operados están satisfechos con los resultados quirúrgicos y mejoran su calidad de vida.

Palabras clave: Hiperhidrosis primaria, simpatectomía, calidad de vida.

\section{Introducción}

La hiperhidrosis es una condición caracterizada por sudoración excesiva que supera las necesidades fisiológicas para mantener la homeostasis térmica del cuerpo ${ }^{1}$. Afecta entre el $1-3 \%$ de la población general, sin diferencia entre sexos ${ }^{2,3}$.

Según la Clasificación Estadística Internacional de Enfermedades (CIE-10), la hiperhidrosis (R61) se puede clasificar en dos entidades principales: hiperhidrosis localizada o primaria (R61.0), que afecta cara y miembros superiores y es provocada por una disfunción del sistema nervioso autónomo simpático ${ }^{4}$, y la hiperhidrosis generalizada o secundaria (R61.1) que constituye más bien un signo que se manifiesta en variadas patologías médicas como, por ejemplo, el hipertiroidismo.

En la hiperhidrosis primaria el exceso de sudoración se manifiesta principalmente en la cara, palmas, axilas y pies. Esto genera una condición muy incómoda para los pacientes, desde la vergüenza en la exposición social, o a lo incapacitante en el ámbito laboral, provocando menoscabo en las relaciones sociales y emocionales, que afecta de manera importante la calidad de vida de los pacientes ${ }^{5}$.

El tratamiento de esta patología puede ser médico o quirúrgico, sin embargo, este último ofrece mejores resultados, sostenidos en el tiempo y con una tasa de satisfacción mayor que el tratamiento médico ${ }^{6}$.

La técnica quirúrgica fue desarrollada por Adson y Brown en 1932, sin embargo, su auge se produjo durante la década de los noventa, cuando se desarrolló la videotoracoscopia, permitiendo un acceso mínimamente invasivo, con escasas complicaciones y secuelas, y con una tasa de éxito de entre $80 \%$ a $100 \%{ }^{7-9}$. A pesar de que muchos grupos quirúrgicos han publicado sus resultados, son pocos los estudios que se han realizado para evaluar los resultados en términos de calidad de vida.

La Organización Mundial de la Salud define calidad de vida como la percepción que un individuo tiene de su lugar en la existencia, en el contexto de la cultura y del sistema de valores en los que vive y en relación con sus objetivos, expectativas, normas e inquietudes. El objetivo de este trabajo es evaluar el cambio en la calidad de vida de los pacientes con hiperhidrosis primaria sometidos a simpatectomía videotoracoscópica.

\section{Material y Método}

Estudio de tipo transversal que incluyó el total de pacientes sometidos a simpatectomía videotoracoscópica en el Hospital FACh entre julio de 2004 y agosto de 2010. Los criterios de inclusión fueron: diagnóstico de hiperhidrosis primaria, mayores de 15 años, beneficiarios de la Fuerza Aérea de Chile y con evaluación dermatológica preoperatoria. Se excluyeron los pacientes con antecedentes psiquiátricos de tipo psicóticos y a quienes no se pudo contactar para ser sometidos a las encuestas.

Los antecedentes epidemiológicos, clínicos y quirúrgicos se obtuvieron del registro de fichas clínicas, previo consentimiento informado. Para evaluar calidad de vida se aplicó un cuestionario estandarizado. En la evaluación de severidad de hiperhidrosis se utilizó la escala HDSS ${ }^{3}$, que realiza una valoración pre y postoperatoria de la tolerancia del paciente frente a su condición y cómo impacta sus actividades cotidianas, graduándola de I a IV. Para el análisis de calidad de vida se utilizó la encuesta de RibasMilanez (2003) ${ }^{7}$, que evalúa aspectos funcionales, personales, sociales y emocionales, otorgándoles una puntuación cuya sumatoria entrega una valoración de la calidad de vida pre y postoperatoria. El desarrollo de sudoración compensatoria se estableció mediante un cuestionario elaborado específicamente para este trabajo, que evalúa el desarrollo postoperatorio de sudoración en zonas del cuerpo que no presentaban este problema en el preoperatorio, además de su localización corporal y una graduación subjetiva del paciente en leve, moderada y severa. El grado de satisfacción se determinó a través de la valoración de los pacientes en cuanto a los resultados obtenidos con la cirugía, graduándola en cinco niveles desde totalmente satisfecho a insatisfecho.

Para el análisis estadístico se utilizó el software SPSS 11.5. 


\section{Resultados}

Entre julio de 2004 y agosto de 2010 se operaron 66 pacientes de simpatectomía videotoracoscópica en el Hospital FACh. Cinco no pudieron ser contactados para la aplicación de las encuestas, reduciendo la muestra a 61 pacientes. El seguimiento promedio fue de 28,6 meses. El promedio de edad fue 25 años (IC 95\%: 22,5-28,3). El 57\% eran de sexo femenino. El IMC promedio fue 22 (IC 95\%: 20-23,9).

El $35,1 \%$ de los pacientes presentaba hiperhidrosis en un segmento corporal, mientras el $64,9 \%$ restante tenía compromiso de dos o más, siguiendo una distribución porcentual según se detalla en la Tabla 1.

En cuanto a la severidad de la hiperhidrosis preoperatorio, el 83\% de los pacientes señaló tener una sudoración apenas tolerable o intolerable, asociado a una gran limitación de las actividades de vida co-

Tabla 1. Localización hiperhidrosis

\begin{tabular}{|lll|}
\hline & n & \% \\
\hline Facial & 11 & 18 \\
Axilar & 39 & 64 \\
Palmar & 42 & 69 \\
Plantar & 9 & 15 \\
\hline
\end{tabular}

tidiana. Este hecho se relaciona directamente con la calidad de vida, siendo ésta pobre o muy pobre en el $82 \%$ de los pacientes. Tablas 2 y 3.

Se utilizó la misma técnica quirúrgica en los 61 pacientes. La duración promedio del procedimiento fue 30 minutos (20-50 min). Todos los pacientes tuvieron una estadía hospitalaria de 1 día. Dos pacientes presentaron complicaciones postoperatorias. Hubo 1 caso de hematoma de pared, que fue manejado en forma conservadora y 1 caso de síndrome de Claude Bernard Horner transitorio, con remisión completa a los 6 meses. Se presentaron 3 casos de recurrencia, todos fueron reoperados, encontrándose la presencia de reinervación de la cadena simpática en 2 pacientes y de un nervio accesorio de Kuntz en 1 paciente. No hubo mortalidad en esta serie.

Como se señaló anteriormente en cuanto a severidad de hiperhidrosis, el 83\% los pacientes presentaban una sudoración apenas tolerable o intolerable en el preoperatorio, sin embargo, en el postoperatorio el 85\% de los pacientes señaló que la sudoración era imperceptible o apenas perceptible y tolerable, y sin repercusión sobre las actividades de la vida cotidiana. Al comparar los períodos pre y postoperatorios la calidad de vida varió de pobre o muy pobre en el $82 \%$ de los pacientes, a mejor o mucho mejor $84 \%$ de éstos. Tablas 4 y 5.

El 78\% de los pacientes operados desarrolló sudoración compensatoria, la que afectaba a más de un segmento corporal en el $83 \%$ de los casos. $14,6 \%$ de

Tabla 2. Escala de severidad de la hiperhidrosis

\begin{tabular}{|llcr|}
\hline Grado severidad & n & \% \\
\hline I & Mi transpiración nunca se notaba y nunca interfería con mis actividades diarias & 1 & 1,6 \\
II & Mi transpiración era tolerable, pero a veces interfería con mis actividades diarias & 10 & 16,3 \\
\hline III & Mi transpiración era apenas tolerable y frecuentemente interfería con mis actividades diarias & 22 & 36,1 \\
IV & Mi transpiración era intolerable y siempre interfería con mis actividades diarias & 28 & 45,9 \\
\hline
\end{tabular}

Tabla 3. Calidad de vida preoperatoria

\begin{tabular}{|lcc|}
\hline \multicolumn{1}{|c|}{$\mathbf{n}$} & $\mathbf{\%}$ \\
\hline Excelente & 0 & 0 \\
Muy buena & 2 & 3,2 \\
Buena & 9 & 14,8 \\
Pobre & 33 & 54,1 \\
Muy pobre & 17 & 27,9 \\
\hline
\end{tabular}

Escala de Ribas-Milanez.
Tabla 4. Comparación de severidad de hiperhidrosis

\begin{tabular}{|lcccc|}
\hline Grado & \multicolumn{2}{c}{ Preoperatorio } & \multicolumn{2}{c|}{ Postoperatorio } \\
severidad & n & \% & n & \% \\
I & 1 & 1,6 & 18 & 29,5 \\
II & 10 & 16,3 & 34 & 55,7 \\
III & 22 & 36,1 & 4 & 6,5 \\
IV & 28 & 45,9 & 5 & 8,2 \\
\hline
\end{tabular}

Escala de Severidad HDSS. Las diferencias entre ambos puntajes son significativas con un $\mathrm{p}=0,001$. 
CALIDAD DE VIDA EN PACIENTES CON HIPERHIDROSIS PRIMARIA SOMETIDOS A SIMPATECTOMÍA ...

Tabla 5. Comparación de calidad de vida

\begin{tabular}{|lcclccc|}
\hline & \multicolumn{2}{c}{ Preoperatorio } & & \multicolumn{2}{c|}{ Postoperatorio } \\
& n & \% & & n & \% \\
Excelente & 0 & 0 & Mucho mejor & 25 & 41 \\
Muy buena & 2 & 3,2 & Mejor & 26 & 42,6 \\
Buena & 9 & 14,8 & Igual & 9 & 14,7 \\
Pobre & 33 & 54,1 & Peor & 1 & 1,6 \\
Muy pobre & 17 & 27,9 & Mucho peor & 0 & 0 \\
\hline
\end{tabular}

Escala de Ribas-Milanez. La diferencia entre el puntaje medio pre y postoperatorio tiene un $\mathrm{p}$ value $<0,05$.

los pacientes la reportaron como leve, 70,8\% como moderada y 14,6\% como severa. La distribución porcentual según segmento corporal afectado se detalla en la Tabla 6.

El 88\% de los pacientes señalaron estar satisfechos con los resultados de la cirugía, comparando su estado de salud preoperatorio y postoperatorio. Tabla 7.

\section{Discusión}

La hiperhidrosis primaria es una condición que altera de manera significativa los aspectos emocionales, sociales y laborales de las personas que la padecen, generando un importante menoscabo en la calidad de vida ${ }^{10}$. La simpatectomía por videotoracoscopia permite un abordaje seguro y mínimamente invasivo. Uno de los principales objetivos que debe perseguir el tratamiento de esta patología es la mejoría en la calidad de vida. Esta última tiene cada vez más importancia en medicina, ya que actualmente el aspecto sicosocial de la vida de los pacientes es relevante al momento de definir manejos terapéuticos. En este estudio se estableció que aproximadamente un $80 \%$ de los pacientes que sufren de hiperhidrosis primaria tienen una mala calidad de vida preoperatoria y su problema de sudoración afecta frecuentemente sus actividades cotidianas. Sin embargo, luego de la simpatectomía, el 83\% mejora su calidad de vida y la sudoración deja de ser una limitación para la realización de las actividades de la vida diaria. El 15\% de los pacientes no mejoró su calidad de vida y hubo 1 paciente que empeoró, operado por rubor facial, que desarrolló sudoración compensatoria severa. La mejoría en la calidad de vida es concordante con lo reportado en la literatura, donde a fines de los años noventa aparece el primer estudio que utilizando el cuestionario SF-36 demostró este cambio $^{11}$. A partir de entonces se realizaron otros trabajos, que utilizando diferentes instrumentos de medición, como por ejemplo el Índice Dermatológico de Calidad de Vida (DLQI), la Escala de Severidad de la Hiperhidrosis (HDSS) y la Encuesta de Ribas-Milanez, demostraron mejoría en términos de calidad de vida, entre un 85 y $95 \%$ de los pacientes operados a través de esta técnica ${ }^{7,12,13}$. Este último instrumento
Tabla 6. Distribución corporal

\begin{tabular}{|lrc|}
\hline Sudoración compensatoria & n & \% \\
Espalda & 41 & 67,2 \\
Tórax & 25 & 40,9 \\
Abdomen & 24 & 39,3 \\
Reg. Inguinal & 12 & 19,6 \\
Rodillas & 11 & 18,1 \\
Piernas & 8 & 13,3 \\
Pies & 7 & 11,4 \\
Muslos & 3 & 4,9 \\
\hline
\end{tabular}

Tabla 7. Grado de satisfacción

\begin{tabular}{|lrr|}
\hline & n & \% \\
\hline Totalmente satisfecho & 9 & 14,7 \\
Bastante satisfecho & 31 & 50,8 \\
Satisfecho & 14 & 22,9 \\
\hline Poco satisfecho & 4 & 6,5 \\
Insatisfecho & 3 & 4,9 \\
\hline
\end{tabular}

fue elegido para este estudio, dado que se trata de una encuesta específica que evalúa todos los aspectos básicos de la calidad de vida en términos de cómo se ven influidos por la hiperhidrosis, realizando además una comparación entre el período pre y postoperatorio.

La morbilidad asociada a este procedimiento ha sido reportada en múltiples publicaciones, siendo en general entre $3-10 \%{ }^{14,15}$. La complicación más frecuentemente observada es la presencia de neumotórax residual que requiere de pleurostomía, que está presente entre el 2 y 14\% de las series. El síndrome de Claude Bernard Horner es una complicación poco frecuente, pero mal tolerada. Se debe a la lesión térmica del ganglio estrellado que se encuentra sobre $\mathrm{T} 1$, desde donde emergen las eferencias nerviosas que controlan el músculo elevador del párpado y ciliar del ojo. Esta complicación se presenta entre el 0,5 y $2 \%$ en la mayoría de las series ${ }^{5,7,9,14-17}$. En la nuestra hubo 1 caso, que remitió en forma espontánea a los 6 meses; lo que se explicaría porque no se seccionó este ganglio, sino más bien por un fenómeno 
de neuropraxia por trasmisión térmica. Para evitar esta complicación se debe limitar la disección con gancho monopolar y se debe realizar la sección de la cadena con tijeras o un bisturí armónico. Nuestra escasa morbilidad tiene que ver con la ejecución de la técnica por un cirujano entrenado en cirugía videotoracoscópica, que realiza el procedimiento en forma estandarizada. No obstante, se debe señalar que nuestro centro concentra poco volumen de pacientes, por lo que es probable que a medida que éste aumente, nuestras complicaciones se equiparen a la de los centros de gran volumen de pacientes.

La persistencia y/o recurrencia de la hiperhidrosis post simpatectomía es un hecho que está pobremente documentado en la literatura, por lo que es difícil estimar su real incidencia. Existen trabajos de los últimos 7 años que presentan tasas de $10 \%$ aproximadamente $^{7,18}$. En 1998 se describió el fenómeno de la reinervación de la cadena como causa de falla ${ }^{19}$, sin embargo, el resto de la literatura ha demostrado que la recurrencia tiene que ver principalmente con errores técnicos, por mala localización de la cadena o sección incompleta de ésta ${ }^{20,21}$. Otro elemento a considerar es la presencia del nervio accesorio de Kuntz, presente en el $10 \%$ de los $\operatorname{casos}^{22}$. Si en estos pacientes no se identifica y secciona este elemento, persiste la comunicación entre los ganglios simpáticos torácicos superiores y el plexo braquial, por lo que no se interrumpe el estímulo simpático a los miembros superiores. La adecuada disección, identificación y sección de la cadena, así como del nervio accesorio de Kuntz, debiera ser la premisa para disminuir la tasa de falla, lo que es relevante teniendo en cuenta que las reintervenciones se asocian a mayores dificultades técnicas y mayor desarrollo de sudoración compensatoria ${ }^{18}$.

El principal problema de la simpatectomía es la sudoración compensatoria, cuyo reporte en la literatura oscila entre el 60 a $90 \%$ de los paciente $^{5,23}$. Nuestra serie presenta una tasa de sudoración compensatoria de $78 \%$, afectando principalmente el tronco, y siendo catalogada por los pacientes como moderada en el $70 \%$ de los casos y severa en el 15\%. La etiología de este fenómeno no está claramente dilucidada, sin embargo, se explicaría a través de un mecanismo de feedback similar al que controla los procesos hormonales. La sudoración excesiva en la hiperhidrosis primaria es desencadenada porque algún estímulo estresante activa los centros de sudoración en el hipotálamo, lo que genera una señal que se transmite por la cadena simpática hacia los órganos efectores (glándulas sudoríparas). Desde los órganos efectores, y por medio de la misma cadena simpática, se envía una respuesta, a través de vías aferentes, hacia el hipotálamo, que constituye una señal de retroalimentación negativo, llevando a esta estructura a cesar su activación, y así a detener la sudoración. Al estar seccionada la cadena, se interrumpiría la vía aferente y los órganos efectores del sistema simpático no podrían enviar la respuesta de retroalimentación negativo, con lo que se perdería la regulación de la sudoración, generando el fenómeno de sudoración compensatoriaa ${ }^{24,25}$. Algunos autores han propuesto que el número de niveles seccionados en la cadena tendría una relación directa con el desarrollo de sudoración compensatoria ${ }^{26}$, sin embargo, en otros trabajos no se ha comprobado esta asociación ${ }^{27-29}$. En nuestra serie tampoco encontramos relación entre sudoración compensatoria y número de raíces seccionadas. Actualmente, se desarrolla una línea de investigación que busca disminuir este efecto mediante el bloqueo de la cadena simpática utilizando clips y evitando así la sección, lo que ha demostrado buenos resultados además de ser reversible ${ }^{24}$.

La relación entre la mejoría de la calidad de vida y el desarrollo de sudoración compensatoria, determinan el grado de satisfacción de los pacientes. En esta serie, aproximadamente un $87 \%$ de los pacientes se manifestó como satisfecho por los resultados de la cirugía, pese al desarrollo de sudoración compensatoria. El 13\% restante se manifestó insatisfecho, en todos los casos producto del desarrollo de este efecto secundario. Este fenómeno es particularmente mal tolerado en el caso de los pacientes que se operan por rubor facial, quienes no sufren de sudoración excesiva preoperatoria. Probablemente la alta tasa de satisfacción tiene que ver con que la sudoración compensatoria afecta zonas del cuerpo que no generan alteración en el desempeño social y laboral de los pacientes, lo que sí ocurría previo a la cirugía. Por lo anteriormente señalado es fundamental que antes de la cirugía, el cirujano tratante discuta con sus pacientes los resultados esperados, así como sus efectos secundarios, de manera de lograr buenos resultados, mejoría de la calidad de vida y satisfacción.

\section{Conclusión}

La hiperhidrosis primaria afecta de manera significativa la calidad de vida de los pacientes que la padecen, generando limitación y menoscabo en el desempeño social, laboral, emocional y físico. El tratamiento quirúrgico mediante la simpatectomía videotoracoscópica ofrece excelentes resultados que son medibles en términos de mejoría de la calidad de vida, con una baja tasa de complicaciones, pero asociado frecuentemente al fenómeno de sudoración compensatoria. Pese al desarrollo de esta secuela, la mayoría de los pacientes operados con esta técnica 
están satisfechos con los resultados quirúrgicos y mejoran su calidad de vida.

\section{Referencias}

1. Athins JL, Butler PE. Hyperhidrosis: a review of current management. Plast Reconstr Surg. 2002;110:222-8.

2. Adar R, Kurchin A, Zweig A, Mozes M. Palmar Hyperhidrosis and its surgical treatment: a report of 100 cases. Ann Surg. 1977;186:34-41.

3. Strutton DR. Kowalski J, Glasser DA, Stang P. US prevalence of hyperhidrosis: results from a national consumer panel. Poster presentation at the Annual Meeting of the American Academy of Dermatology. March 21-26, 2003; San Francisco, Calif. Poster abstract P362.

4. Santolaya R. Simpatectomía torácica en la hiperhidrosis primaria. Cuad Cir. 2006;20:86-91.

5. Dewey T, Herbert M, Hill S, Prince S, Mack M. One year follow up after thoracoscopic sympathectomy for hyperhidrosis: Outcomes and consequences. Ann Thorac Surg. 2006;81:1227-33.

6. Ambrogi V, Campione E, Mineo D, Paterno E, Pompeo E, Mineo T. Bilateral thoracoscopic T2 to T3 sympathectomy versus botulinum inyection in palmar hyperhidrosis. Ann Thorac Surg. 2009;88:238-45.

7. Ribas Milanez JR, Kauffman P, De Campo G, Oliveira L, Kusniek S, Wolosker N. Quality of life, before and after thoracic sympathectomy: report on 378 operated patients. Ann Thorac Surg. 2003;76:886-98.

8. Haider A, Solish, N. Focal Hyperhidrosis: Diagnosis and treatment. CMAJ. 2005;172:69-75.

9. Xu L, Yuan T, Min L, Fan L, Jiang C, Zhujian D. Endoscopic Thoracic Sympathectomy for Palmar Hyperhidrosis: A Randomized Control Trail Comparing T3 and T2-T4 Ablation. Ann Thorac Surg. 2008;85:1747-52.

10. Kumagai K, Kawase H, Kawanishi M. Health related quality of life after thoracoscopic sympathectomy for palmar hyperhidrosis. Ann Thorac Surg. 2005;80:461-6.

11. Sayeed R, Nyamekye I, Ghana S, Poskitnik S. Quality of Life after transthoracic endoscopic sympathectomy for upper limb hyperhidrosis. Eur J Surg. 1998;Suppl 580:39-42.

12. Loscertales J, Arroyo T, Congregado M, Jiménez R, Girón J, Arenas C, y cols. Tratamiento de la Hiperhidrosis palmar por simpatectomía torácica. Resultados inmediatos y calidad de vida postoperatoria. Arch Bronconeumol. 2004;40:67-71.

13. Boley T, Belangee K, Markwell S, Hazelrigg S. The effect of thoracoscopic sympathectomy on quality of life and symptoms management of hiperhidrosis. J Am Coll Surg. 2007;204;3:435-8.

14. Herbst F, Plas E, Fugger R, Fritsch A. Endoscopic thoracic sympathectomy for primary hyperhidrosis of the upper limbs. A critical analysis and long-term results of 480 operations. Ann Surg. 1994;220:86-90.
15. Schmidt J, Bechara F, Altmeyer P, Zirngib L. Endoscopic thoracic sympathectomy for severe hyperhidrosis: impact of restrictive denervation on compensatory sweating. Ann Thorac Surg. 2006;81:1048-55.

16. Doolabh, N, Horswell S, Williams M, Huber L, Prince $\mathrm{S}$, Meyer D, et al. Thoracoscopic sympathectomy for hyperhidrosis: indications and results. Ann Thorac Surg. 2004;77:410-4.

17. Kwong F, Hobbs J, Cooper L, Burrows W, Gamliel Z, Krasna M. Clinical Experience in 397 consecutive thoracoscopic sympathectomies. Ann Thorac Surg. 2005;80:1063-6.

18. Freeman R, Van Woerkom, J, Vyverberg A, Ascioti A. Reoperative endoscopic sympathectomy for persistent or recurrent palmar hyperhidrosis. Ann Thorac Surg. 2009;88:412-7.

19. Singh B, Moodley J, Wafferjee A, Ramidal P, Robbs J, Rajaruthnam P. Resympathectomy for sympathetic regeneration. Surg Laparosc Endosc. 1998;8:257-60.

20. Orteu CH, McGregor JM, Almeyda JR. Recurrence of hyperhidrosis after endoscopic transthoracic sympathectomy: case report and review of the literature. Clin Exp Dermatol. 1995;20:230-3.

21. Lin TS. Video-assisted thoracoscopic resympathectomy for palmar hyperhidrosis. Analysis of 42 cases. Ann Thorac Surg. 2001;72:895-8.

22. Kuntz A. Distribution of the sympathetic rami to the braquial plexus. Arch Surg. 1927;15:871-7.

23. Lin TS, Fang HY. Transthoracic endoscopic sympathectomy in the treatment of palmar hyperhidrosis with enphasis on perioperative management (1360 case analyzed). Surg Neurol. 1999;52:453-7.

24. Neumayer C, Zacherl J, Holak G, Fugger R, Jakesk R, Herbst F, et al. Limited endoscopic thoracic sympathetic block for hyperhidrosis of the upper limb. Surg Endosc. 2004;18:152-6.

25. Chou SH, Kao E, Lin C, Chang Y, Huang M. The importance of classification in sympathetic surgery and a proposed mechanism for compensatory hyperhidrosis. Experience with 464 cases. Surg Endosc. 2006;20:174953.

26. Hederman WP. Present and future trends in thoracoscopic sympathectomy. Eur J Surg. 1994;572 (Suppl):17-9.

27. Moya J, Ramos R, Vives N, Pérez J, Morera R, Perna $\mathrm{V}$, et al. Compensatory sweating after upper thoracic sympathectomy. Prospective study of 123 cases. Arch Bronconeumol. 2004;40:360-3.

28. Lai YT, Yang LH, Chio CC. Complications in patients with palmar hyperhidrosis treated with transthoracic endoscopic sympathectomy. Neurosurgery 1997;41:110-3.

29. Lesèche $G$, Lastier $Y$, Thabut $G$, Petit $M$, Combes $M$, Green O, et al. Endoscopic transthoracic sympathectomy for upper limb hyperhidrosis: limited sympathectomy does not reduce postoperative compensatory sweating. J Vasc Surg. 2003;37:124-8. 